\title{
Disentangling depression in Belgian higher education students amidst the first COVID- 19 lockdown (April-May 2020)
}

Jeroen De Man ${ }^{1 *}$ (D, Veerle Buffel ${ }^{2}$, Sarah van de Velde ${ }^{2}$, Piet Bracke ${ }^{3}$, Guido F. Van Hal ${ }^{4}$, Edwin Wouters ${ }^{2}$ and for the Belgian COVID-19 International Student Well-being Study (C19 ISWS) team

\begin{abstract}
Background: The surge of COVID-19 infections has prompted many countries to take unprecedented policy measures. In Belgium, the authorities implemented a nation-wide stay-at-home order for several months. Evidence of the mental health effect of such measures is scarce. A recent review by Brooks et al. has compiled a defined list of stressors affecting people's mental health under quarantine during previous epidemic settings. This study aims to test the association between these stressors and the mental health of students attending higher education during the stay-at-home order in Belgium.

Methods: In this cross-sectional study, 18,301 students from 13 higher education institutions (HEl) participated in an online survey between 26 April and 11 May 2020. We assessed the association between potential stressors and depressive symptoms severity scores and structural equation modeling was used to assess how stressors may mediate the association between duration of exposure and depressive symptoms severity.

Results: The stressors proposed by Brooks et al. were found to be associated with depressive symptoms severity. The stressors 'perceived academic stress', 'institutional dissatisfaction' and 'fear of being infected' were associated with substantially higher depressive symptoms severity scores. The association between duration of exposure and depressive symptoms severity was mediated by 'academic stress'. Being in a steady relationship and living together with others were both associated with a lower depressive symptoms severity.
\end{abstract}

Conclusion: Findings underline the need for a student-centered approach and mental health prevention. Authorities and HEls should consider whether and if so, how a stay-at-home order should be implemented.

Keywords: COVID-19, Stay-at-home order, Belgium, Mental health, Higher education students, Depressive symptoms, Academic stress, Fear of infection, Institutional dissatisfaction

\footnotetext{
* Correspondence: jeroen.deman@uantwerpen.be

'Department of Family Medicine and Population Health, University of

Antwerp, Doornstraat 331, 2610 Wilrijk, Belgium

Full list of author information is available at the end of the article
}

(c) The Author(s). 2021 Open Access This article is licensed under a Creative Commons Attribution 4.0 International License, which permits use, sharing, adaptation, distribution and reproduction in any medium or format, as long as you give appropriate credit to the original author(s) and the source, provide a link to the Creative Commons licence, and indicate if changes were made. The images or other third party material in this article are included in the article's Creative Commons licence, unless indicated otherwise in a credit line to the material. If material is not included in the article's Creative Commons licence and your intended use is not permitted by statutory regulation or exceeds the permitted use, you will need to obtain permission directly from the copyright holder. To view a copy of this licence, visit http://creativecommons.org/licenses/by/4.0/. The Creative Commons Public Domain Dedication waiver (http://creativecommons.org/publicdomain/zero/1.0/) applies to the data made available in this article, unless otherwise stated in a credit line to the data. 


\section{Background}

The COVID-19 pandemic has had a profound impact on global health, with, as of September 2020, more than 27 million confirmed cases and almost 900,000 deaths [1]. Belgium was hit hard by the pandemic: by September 2020, authorities reported more than 88,000 confirmed cases and almost 10,000 deaths. Transmission of COVID-19 within the country was confirmed in early March 2020 and increased rapidly during March and April 2020. Belgium was among the world's worstaffected countries in terms of the number of deaths per capita attributed to COVID-19, although this was likely due to using a different method of reporting compared to other countries [2].

The rapid spread of the virus spurred a series of unprecedented policy measures, including a stay-at-home order. On 18 March, strict physical distancing measures were imposed, with non-essential travel prohibited [3]. People were only allowed to leave their house to buy food or go to work if considered essential (e.g., people working in healthcare, transportation, food distribution, etc.). All bars, restaurants and shops providing nonessential services were closed, but individual physical exercising was still allowed. Gatherings were banned, and students were prohibited to attend classes physically and higher education institutions (HEI) were forced to move to online teaching methods. Penalties were imposed for individuals who failed to comply [3].

The implementation of such radical measures comes with multiple side-effects, and experts expressed their concern about a profound impact on mental health [4]. However, direct physical consequences (i.e., morbidity and mortality directly caused by COVID-19) have typically been given more weight in decision-making and public health interventions. An example of this was the suspension of schools in 188 countries, affecting over $90 \%$ of enrolled students globally (1.5 billion young people) [5], despite conflicting evidence of a substantial contribution to COVID-19's transmission control [6]. To obtain a more balanced image, mental health experts have been calling for immediate and high-quality evidence on the mental health impact of the COVID-19 pandemic and related containment measures from novel population-based studies [4]. In addition, experts reached a consensus on the urgent need to identify the mechanisms that are affecting mental health during the pandemic in order to provide evidence-based and mechanistically informed psychological treatment and public health interventions [4].

College and university students have been shown a vulnerable group regarding mental health. Mental disorders have been shown common in this population and are typically untreated [7]. A German study reported a higher prevalence of depressive symptoms in university students compared to the general population, which the authors attributed to an increase in academic demand and a decrease in peer support [8]. During the COVID19 pandemic, students have been confronted with a combination of government and institutional specific measures - potentially adding to their mental health vulnerability [5]: 1) a radical transformation from in-person to online teaching and evaluation; 2) cancellations of anticipated events such as exchange studies, internships, graduation ceremonies, etc.; 3 ) the loss of part-time jobs; and 4) a shrinking job market enhancing uncertainty among students in their final year.

Preliminary evidence from studies conducted during the current pandemic indeed seems to suggest their age group to be among the most affected [9-12]. Moreover, a study in 1000 university students in Greece reported an alarming increase in suicidal thoughts and symptoms severity scores of depression and anxiety during the first phase of a national stay-at-home order [13]. A study in 7143 medical students in China reported a high level of anxiety and found an association with COVID-19related stressors such as economic stressors, effects on daily-life, and academic delays [14]. A study with university students in Spain also reported a negative effect on their mental health [15]. However, it remains unclear why and how this subgroups' mental health has been affected, and several potential stressors remain unexplored.

A recent review identified a comprehensive set of mental health stressors that were shown to play a role in specific populations of infected people who were quarantined to limit the transmission of pathogens similar to COVID-19 [16]. These stressors included: frustration and boredom, inadequate supplies of resources, inadequate information from public health authorities, insufficient financial resources, perceived stigma, and fears of infection. Duration of the exposure to quarantine was also found to play a role, but it was unclear through which mechanism.

We hypothesize that the same type of mental health stressors, after contextualization, could play a role among students attending higher education exposed to a stay-at-home order during the COVID-19 pandemic. Contextualization of these stressors relates to the study population (i.e., higher education students) and to the exposure (i.e., a stay-at-home order vs. quarantine). For example, tailored to this study population of students, we propose to examine how COVID-19 related repercussions on their academic work (i.e., academic stress) and their institutions' response to the COVID-19 pandemic (i.e., institutional dissatisfaction) are related to the mental health of these students. Of this compilation of stressors, we further hypothesize that the level of boredom and academic stress may typically worsen over a 
relatively short time period (i.e., 2 weeks). As such, we think that these two stressors may explain the assumed association between duration of exposure to the stay-athome and mental health outcomes.

This study aims to investigate the association between the COVID-19 pandemic - and related containment measures - and students' mental health attending higher education in Belgium. In particular, we will examine the association between depressive severity scores and a set of mental health stressors identified by Brooks et al. during quarantine, after tailoring them to our study population. Finally, we will test whether the association between the duration of the stay-at-home order and students' mental health is mediated through the stressors' perceived boredom' and 'perceived academic stress'.

\section{Methods}

\section{Data-collection and procedures}

In order to address the above-cited research aims, the study employed a structured questionnaire administred to a convencience sample of students: a link to an online survey was sent by e-mail to the students of 13 higher education institutions (HEI) located in Belgium. Participants were eligible if they were enrolled in a higher education program, aged 18 or above, and provided informed consent. Data collection took place between 26 and 04-2020 and 11-05-2020 corresponding to day 39 and 54 after the stay-at-home order started. Twenty-five thousand two hundred seventy-two students started the survey and 21,270 students completed it. The study population was further reduced to 18,301 after applying additional selection criteria: age below 31 years, enrolled in a bachelor's or master's program, residing in Belgium during the pandemic and not having been diagnosed with COVID-19 at the time of the study. The latter group was not included to avoid interference with the stressor 'fear of infection'.

The study is part of the COVID-19 International Student Well-being Study (C19 ISWS) in which 110 HEIs of 26 countries participated. More details about the study procedures can be found in the study protocol [17].

The 8 items of the CES-D scale, used as the response variables, did not have missing values. The percentage of missing data in other variables varied between 0.0 and $0.5 \%$, except for perceived stigma (6.1\%). Multivariate imputation by chained equations with predictive mean matching for continuous data, logistic regression imputation for binary data and polytomous regression imputation for unordered categorical data (factor $>2$ levels) was used to handle the missing data under a missing at random assumption. The procedure was done using the 'Mice' package in R [18]. Rubin's rules were used to pool point and SE estimates across 20 imputed data sets. The results of this approach were compared with the results of a complete case analysis.

\section{Measures}

Standard demographic and socioeconomic questions were asked to determine gender, age, the number of people living with, citizenship, relationship status, and financial ability to cover living expenses before the stayat-home order.

The eight-item version of the Center for Epidemiological Studies Depression Scale (CES-D scale) [19] was used to measure severity of depressive symptoms and has shown adequate psychometric properties [20]. Response options are based on a 4-point Likert scale ranging from 'none or almost none of the time' to 'all or almost all of the time'. We assumed correlated residuals between the positively worded items as reported by others [20].

Potential stressors affecting mental health, as proposed by Brooks et al. [16], were measured mostly with selfdeveloped constructs as they were deemed the most adequate for the specific and novel context of the COVID19 epidemic. These stressors were contextualized to the study population and to the exposure (i.e., a stay-athome order vs. quarantine) as illustrated in Additional file 1. Level of boredom, inadequate supply of resources, perceived pandemic-related stigma and insufficient financial resources were measured by 1 item each. Fear of infection was measured by a 4-item construct. Tailored to the study population, two 4-item constructs were used to measure academic stress and institutional dissatisfaction. Responses were measured through 4, 5, and 11-point Likert scales ranging between 2 extremes, such as 'strongly agree' to 'strongly disagree'. The duration of exposure to stay-at-home order was measured using the date of response submission. More detail on the measures can be found in Additional file 1 and in the study protocol [17].

\section{Data-analysis}

Descriptive statistics were used to provide an overview of participants' answers to essential sociodemographic variables, the outcome and the stressors.

Confirmatory factor analysis was used to assess the measurement models for depressive symptoms severity, academic stress, institutional dissatisfaction and fear of being infected. The objective of confirmatory factor analysis is to test whether data fit a hypothesized measurement model which assigns a value to an unobserved or latent variable based on its' relationship with observed variables. A second-order model was used for fear of being infected since it included two subconstructs: infection of oneself and infection of someone in one's personal network. The following indices of fit and 
related benchmarks for acceptable fit were used [21]: comparative fit index (CFI) $(\geq 0.95)$, Tucker-Lewis index (TLI) $(\geq 0.95)$, root mean square error of approximation (RMSEA) ( $\leq 0.08)$, and standardized root mean square residual (SRMR) ( $\leq 0.08)$. Diagonally weighted least squares was used for parameter estimation as latent constructs were assumed normally distributed.

To test the association between the hypothesized stressors and depressive symptoms severity, the following constructs were regressed on the CES-D scores: boredom, inadequate supply of resources, perceived pandemic-related stigma, insufficient financial resources, inadequate information, fear of infection, fear of significant others being infected, academic stress and institutional dissatisfaction. The following factors were used as control variables: gender, age, the number of people living with, citizenship, being in relationship, and financial ability to cover living expenses before the stay-at-home order. To test the hypothesized mediation through the stressors boredom and academic stress, a structural equation model was used with the CES-D as outcome and duration of the stay-at-home order as independent variable. Criteria for mediation were based on the definition by Cerin [22]: (1) the mediating variable relates to the independent variable; and (2) occurrence of a significant association between the mediating variable and the outcome variable, after adjustment for the independent variable. The fit of the mediation model was assessed based on the same fit indices and benchmarks used for the measurement models. Data were analyzed with $\mathrm{R}$ software and the packages 'lavaan' [23] and 'semTools' [24].

\section{Results \\ Population characteristics}

The study population's mean age was 21 and included a higher proportion of females, bachelor students and natives (see Table 1). More than half of the participants reported to be in a steady relationship and the majority was living together with two or more people. More than $90 \%$ reported having sufficient resources to cover their living expenses.

On average, concern about being infected or severely ill was rated lower than concern about someone in the participants' personal network being infected or severely ill (see Table 2). A majority of students reported sufficient financial resources to cover their expenses. Approximately one third reported to be bored most or all of the time. Ratings of the items related to academic stress indicated an increase of this stressor due to COVID-19 among a high proportion of students. Responses to items related to institutional dissatisfaction were mixed, with some items reflecting satisfaction and others dissatisfaction in a higher proportion of students.

\section{Measurement models}

Item indicators of the constructs measuring depressive symptoms severity, academic stress and institutional dissatisfaction were significant $(\mathrm{z}>1.96)$ and loaded adequately on the latent variables (see Additional file 2). Criteria of fit were met for these constructs.

Table 1 Summary characteristics and depressive symptoms scores of university students in Belgium, April-May 2020 (N = 18,301)

\begin{tabular}{|c|c|c|}
\hline Characteristic & Category & proportion (\%) \\
\hline \multirow[t]{2}{*}{ Age (years) } & mean: 21.0 (SD: 2.32) & \\
\hline & range: $17-30$ & \\
\hline \multirow[t]{2}{*}{ Gender } & Female & 73.9 \\
\hline & Male & 26.1 \\
\hline \multirow[t]{2}{*}{ Citizenship } & Belgian & 91.8 \\
\hline & Foreign & 8.2 \\
\hline \multirow[t]{2}{*}{ Study program } & Bachelor & 75.9 \\
\hline & Masters & 24.1 \\
\hline First-year student & & 22.6 \\
\hline steady relationship & & 47.6 \\
\hline \multirow[t]{3}{*}{$\mathrm{N}^{\circ}$ of people living with (during the stay-at-home order) } & 0 & 3.3 \\
\hline & 1 & 11.6 \\
\hline & 2 or more & 85.1 \\
\hline Insufficient financial resources to cover living expenses (before the stay-at-home order) & & 3.1 \\
\hline \multirow[t]{2}{*}{ CES-D 8 depressive symptoms scores } & mean & 10.8 \\
\hline & standard deviation & 5.08 \\
\hline
\end{tabular}

Legend: CES-D Center for Epidemiological Studies Depression Scale 
Table 2 Descriptive statistics of stressors and related items among university students in Belgium, April-May 2020 (N = 18,301)

\begin{tabular}{l} 
'Stressor' / related items \\
\hline 'Fear of being infected': \\
Concern of being infected \\
Concern of becoming severely ill because of COVID \\
Concern of infection in personal network \\
Concern of someone in personal network becoming severely ill because of COVID-19
\end{tabular}

Category or scale
Statistic mean (SD)

'Concern of health services running out of supplies'

‘level of boredom’

$\begin{array}{ll}\text { none of the time } & 33.5 \\ \text { some of the time } & 36.3 \\ \text { most of the time } & 21.4 \\ \text { all of the time } & 8.8\end{array}$

\section{'COVID-19 related academic stress':}

Increase in academic workload

strongly agree $\quad 35.6$

Course expectations are less clear

Interference with academic performance

agree

neither agree nor disagree

disagree

strongly disagree

strongly agree

agree

neither agree nor disagree

disagree

strongly disagree

agree

neither agree nor disagree

\section{'COVID-19 related institutional (dis)satisfaction':}

Perceived decrease in quality of education

strongly agree
agree
neither agree nor disagree
disagree
strongly disagree
strongly agree
agree
neither agree nor disagree
disagree
strongly disagree
strongly agree


Table 2 Descriptive statistics of stressors and related items among university students in Belgium, April-May 2020 ( N = 18,301) (Continued)

\begin{tabular}{|c|c|c|}
\hline 'Stressor' / related items & Category or scale & Statistic \\
\hline & agree & 42.2 \\
\hline & neither agree nor disagree & 27.5 \\
\hline & disagree & 12.7 \\
\hline & strongly disagree & 5.1 \\
\hline \multirow[t]{5}{*}{ University staff is accessible to share concerns } & strongly agree & 6.8 \\
\hline & agree & 25.5 \\
\hline & neither agree nor disagree & 34.2 \\
\hline & disagree & 21.7 \\
\hline & strongly disagree & 11.8 \\
\hline \multirow[t]{5}{*}{ 'Sufficient financial resources to cover expenses' } & strongly agree & 40.1 \\
\hline & agree & 33.0 \\
\hline & neither agree nor disagree & 12.5 \\
\hline & disagree & 9.6 \\
\hline & strongly disagree & 3.9 \\
\hline 'The participant reported hiding symptoms from others' & & 37.5 \\
\hline
\end{tabular}

\section{Association between stressors and depressive symptoms severity}

Each of the hypothesized stressors was positively associated with depressive symptoms severity independent of their adjustment for the control variables (see Table 3). Stressors associated with a higher score of depressive symptoms severity included 'academic stress', 'institutional dissatisfaction', 'boredom' and 'fear of infection' associated with, respectively, a $0.66,0.51,0.25$, and 0.27 increase in depressive symptoms severity (on a scale from zero to three) per one-unit increase of the stressor. More response units were used to measure the stressors

Table 3 Associations between stay-at-home order related stressors and depressive symptoms severity among university students in Belgium, April-May $2020(N=18,301)$

\begin{tabular}{|c|c|c|c|c|}
\hline variable & scale & Base model & Crude model & Adjusted model \\
\hline & & Estimate (SE) & Estimate (SE) & Estimate (SE) \\
\hline age & yrs. & $-0.030^{* *}(0.003)$ & & $-0.024^{* *}(0.003)$ \\
\hline female gender & $0-1$ & $0.290 * *(0.016)$ & & $0.284^{* *}(0.016)$ \\
\hline non-Belgian citizenship & $0-1$ & $0.148^{* *}(0.027)$ & & $0.009(0.026)$ \\
\hline having a steady relationship & $0-1$ & $-0.118^{* *}(0.015)$ & & $-0.119^{* *}(0.014)$ \\
\hline living with 1 person ${ }^{a}$ & $0-1$ & $-0.249^{* *}(0.044)$ & & $-0.219^{* *}(0.043)$ \\
\hline living with 2 or more people ${ }^{a}$ & $0-1$ & $-0.267^{* *}(0.040)$ & & $-0.217^{* *}(0.039)$ \\
\hline Insufficient financial resources (before the stay-at-home order) & $0-1$ & $0.179^{* *}(0.009)$ & & $0.025^{*}(0.011)$ \\
\hline fear of infection & $0-10$ & & $0.281^{* *}(0.013)$ & $0.266^{* *}(0.014)$ \\
\hline level of boredom & $0-3$ & & $0.239^{* *}(0.007)$ & $0.246^{* *}(0.007)$ \\
\hline academic stress & $0-4$ & & $0.655^{* *}(0.012)$ & $0.663^{* *}(0.012)$ \\
\hline insufficient medical supplies & $0-10$ & & $0.055^{* *}(0.002)$ & $0.049^{* *}(0.002)$ \\
\hline institutional dissatisfaction & $0-4$ & & $0.514^{* *}(0.014)$ & $0.512^{* *}(0.014)$ \\
\hline insufficient fin. Resources & $0-4$ & & $0.151^{* *}(0.006)$ & $0.144^{* *}(0.008)$ \\
\hline perceived stigma $^{\mathrm{b}}$ & $0-1$ & & $0.140^{* *}(0.020)$ & $0.118^{* *}(0.020)$ \\
\hline
\end{tabular}

Legend: unstandardized parameter estimates of the association between depressive symptoms severity as a latent construct and the control variables (base model), the hypothesized stressors without control variables (crude model) and the hypothesized stressors with control variables (adjusted model). Fear of infection, academic stress and institutional dissatisfaction represent latent constructs. Parameter estimates correspond to the effect on depressive severity on a scale from 0 to 3 ('none of the time' to 'all of the time') per 1-unit change of the predictor. ${ }^{a}$ reference: living with no other people. ${ }^{b}$ estimates of perceived stigma were based on a subsample of participants reporting symptoms that could potentially evoke stigma. $P$-value codes: $p<0.05{ }^{\prime * \prime}, p<0.001{ }^{\prime * * \prime}$ 
'insufficient medical supplies at health services' and 'fear of infection' which implies that, in case of equal estimates, the effect size between two extreme response positions will be higher compared to stressors measured with less response units.

All controlling variables showed a significant association with depressive symptoms severity in the base model. In the adjusted model, the association with nonBelgian citizenship and insufficient financial resources (before the stay-at-home order) disappeared or weakened, suggesting their association being explained by one of the stressors. Sensitivity analysis based on complete case analysis produced similar results.

\section{Mediation path model}

Per extra day of exposure to the stay-at-home order, average scores of depressive symptoms severity were 0.009 higher (i.e. the total association between duration of exposure and depressive symptoms severity). This association was partly mediated by academic stress, but not by perceived level of boredom (see Fig. 1). The model showed an acceptable fit $(\mathrm{CFI}=0.943$; $\mathrm{TLI}=0.971$ : RMSEA $=0.060$; SRMR=0.056). Sensitivity analysis based on complete case analysis produced similar results.

\section{Discussion}

This study provides novel insight into the repercussions of a stay-at-home order implemented during the peak of the COVID-19 pandemic on depressive symptoms severity among students attending higher education in Belgium. The aim of this study was to test a contextualized version of the stressors that Brooks et al. identified [16] in specific populations exposed to quarantine. Our findings indicated that those stressors were also relevant in students exposed to a stay-at-home order. The stressors 'academic stress', 'institutional dissatisfaction', and 'fear of infection' were associated with a strong increase in depressive symptoms severity. Duration of the exposure also showed to have an effect on depressive symptoms severity, which was mediated by 'academic stress'. Being in a steady relationship and living together with others were associated with a lower depressive symptoms severity.

To our knowledge, academic stress and institutional dissatisfaction have not been tested as stressors in a similar setting study during the COVID-19 pandemic. The strong association with depressive symptoms severity is in line with studies during a non-epidemic setting showing depression to be highly correlated with perceived study demands [8]. With regards to academic stress, a majority of students indicated an increase in academic workload, course expectations being less clear, being concerned about their academic performance and being distressed because of a change in teaching methods. Regarding institutional dissatisfaction, half of the students reported a decrease in quality of education and one third reported university staff (e.g., a professor, a student counselor, etc) not being accessible to share concerns about COVID-19. However, more than half of the students also reported that their HEI provided sufficient information about changes due to COVID-19 and adequate implementation of protective measures. Overall, these findings are important warning signs for HEI to carefully monitor and address academic demand and for academic staff to assure the quality of teaching and a student-centered approach.

On average, fear of being infected was rated lower than fear of someone from the participants' personal network being infected. This suggests that students are more concerned about significant others than about themselves being infected, which we assume may be due to seeing themselves running a lower risk of

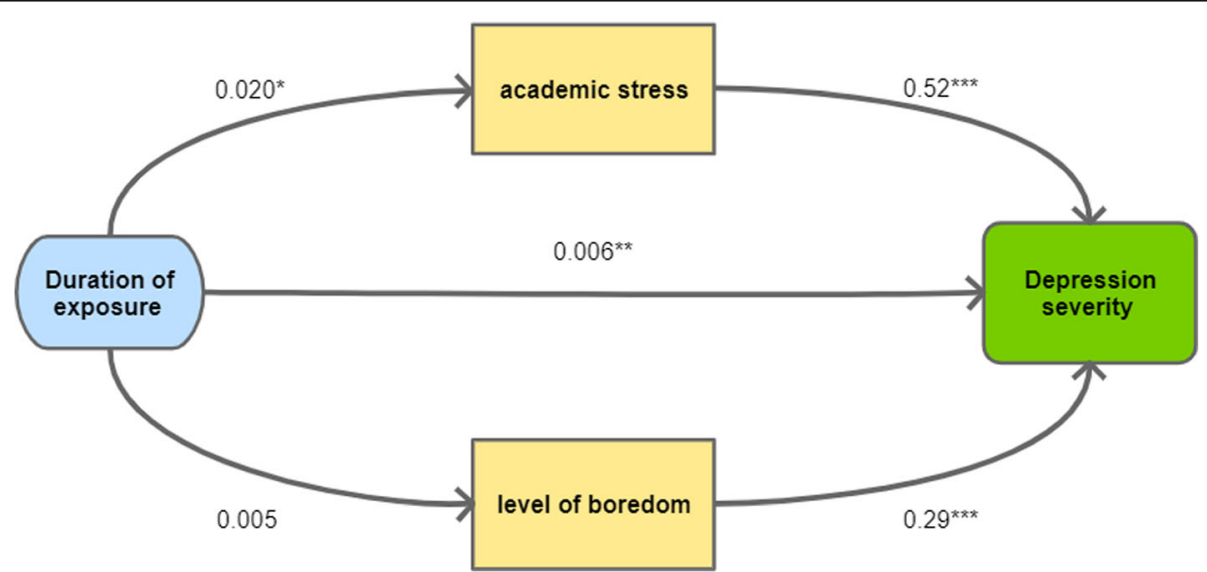

Fig. 1 Mediation model between duration of exposure and depressive symptoms severity among university students in Belgium, April-May 2020 $(N=18,301)$. The estimates are fully standardized and adjusted for the control variables mentioned in methods. Duration of exposure was measured in days. $P$-value codes: $p<0.05^{\prime * \prime}, p<0.01^{\prime * * *^{\prime}}, p<0.001^{{ }^{* * * *^{\prime}}}$ 
complications because of their younger age. Evidence from other studies has been mixed regarding these stressors. Cao et al. found having significant others to be infected by COVID-19 an important risk factor for experienced anxiety among students, potentially due to a higher perceived probability of themselves being infected [14]. However, the latter assumption is in contrast with our findings. A study in a general population in Italy found an effect on depression if an acquaintance was infected by COVID-19, but not if a family member was infected by COVID- 19 [25].

Living together with others and being in a steady relationship were associated with lower depressive symptoms severity scores. We assume this is due to social interaction preventing depressive tendencies through boredom or feelings of loneliness, but they may also relate to social support when one goes through personal challenges or academic stress. Similar associations have been shown in other studies [8], including a study during the COVID-19 pandemic [14].

Perceived stigma among people with COVID-19 related symptoms did not show a strong association with depressive symptoms, while it was found a major theme among people being quarantined [16]. We assume this can be explained by the difference between quarantining and a stay-at-home order, with the latter being applicable to the whole population and not only to a subgroup of infected people. In addition, our study only considered people who were not diagnosed with COVID-19.

Duration of exposure to the stay-at-home order only had a small effect, which was expected since datacollection only started at day 39 of the stay-at-home order. Moreover, the implemented stay-at-home order rather was a light version with, for instance, outdoor physical activity still allowed. The few studies examining the duration of exposure in quarantined individuals [16], reported an effect on other mental health outcomes, but not on depression. The model in our study was in support of the association between duration and depressive symptoms severity being partly mediated by academic stress. Again, this emphasizes the need for monitoring and addressing students concerns and frustrations.

Two of the major strengths of this study include: (1) the large sample of students from 14 HEIs in the height of the Belgian COVID-19 crisis; and (2) data covering the full range of mental health stressors outlined by a recent review study on the mental health consequences of quarantine. This study also has several limitations. Putting our findings into perspective, the big sample size of the study increased the probability of a type- 1 error. The stay-at-home order also needs to be seen as a light version' with several movements still allowed (e.g., outdoor physical activity, commuting, public transport). We also want to remark that it is impossible to know to which extent the associations found in our study can be attributed to the stay-at-home order, direct effects of the pandemic (e.g., mortality), or other causes. For example, the established association with 'fear of being infected' likely depends more on direct COVID-19 related morbidity and mortality than on the stay-at-home order triggered by the pandemic. Another limitation of the study was the use of an online survey which is prone to selfselection bias. Comparing our study sample with governmental data of higher education students enrolled in Flanders in 2018-2019 [26], reveals a higher proportion of females $(+/-20 \%)$ and a similar proportion of foreign nationals. We did not see a decline in values of key variables such as depressive outcome, perceived academic stress and institutional dissatisfaction among students who participated during a later stage of the study, which is in support of sample validity for these key variables. We conclude that based on this convenience sample, we cannot claim estimates of depression status and other variables to be representative of the overall Belgian student population. We think, however, that after controlling for key variables, the reported relationships are reasonable reflections of a general trend. Another limitation concerns the adequacy of the measures being used. Because of a lack of contextually validated constructs the novelty and specific character of the COVID-19 epidemic forced us to be innovative - most of these measures were developed and used for the first time with several one or two-item constructs. Finally, the crosssectional study design did not allow us to examine any longitudinal effects or confirm causal relationships.

\section{Conclusions}

Our findings underline the importance of a studentcentered approach in students exposed to a stay-at-home order during the COVID-19 pandemic. We recommend authorities and HEI to carefully consider whether and if yes, for how long and how strict a stay-at-home order is essential using a multidisciplinary approach. In case such an intervention is required, major stressors such as 'academic stress' and 'institutional dissatisfaction' need to be monitored and addressed by HEI. Concretely, we recommend clear and straightforward communication and to stimulate interactions between students and teachers through realtime teaching. Students may also be encouraged to ensure in-person social interactions (e.g., temporarily move in with parents, family or friends), which were suggested protective. Monitoring and reconsidering academic demand is warranted. We further recommend HEI to monitor students' mental well-being and to offer accessible mental health support, with special attention for students who are prone to social deprivation and who are living alone. Finally, our findings confirm the importance of further research on mental health during pandemic containment measures. 


\section{Supplementary Information}

The online version contains supplementary material available at https://doi. org/10.1186/s13690-020-00522-y.

Additional file 1. Contextualisation of COVID-19 stressors. Additional file 2.

\section{Abbreviations}

CES-D: Center for Epidemiological Studies Depression; CFI: Comparative Fit Index; COVID-19: Coronavirus Disease of 2019; HEl: Higher Education Institutions; TLI: Tucker-Lewis Index; RMSEA: Root Mean Square Error of Approximation; SRMR: Standardized Root Mean square Residual

\section{Acknowledgements}

This study is part of the Belgian COVID-19 International Student Well-being Study (C19 ISWS). The following members are part of the C19 ISWS team: Prof. Sylvie Gadeyne, Department of Sociology, Interface Demography, VUB; Hanne (P.J.) Kindermans, Research group Healthcare and Ethics, Faculty of Medicine and Life Sciences, Hasselt University; Mathilde Joos and team for HOGENT University of Applied Sciences and Arts (Belgium); Sander Vanmaercke, Vlaamse Vereniging van Studenten (WS); Pr. Anne-Sophie Nyssen, Vice Rector of Education and Welfare, ULiège; Ninon Puttaert, researcher - Organisational Psychology - Ergonomics, ULiège; Dries Vervecken, Karel de Grote University College; Marlies Van Guyse and team for LUCA School of Arts (Belgium).

\section{Authors' contributions}

EW, VB and SV played a major role in the conception of the study. EW, VB, GV, PB and SV played a major role in the design of the study. EW, VB, GV, PB and SV organised the data acquisition. JD drafted the manuscript and analyzed and interpreted the data. EW, VB, GV, PB and SV contributed to the interpretation of the data and provided inputs into the writing. All authors read and approved the final manuscript.

\section{Funding}

This work was supported by the Faculty of Medicine and Health Sciences of the University of Antwerp [37025].

\section{Availability of data and materials}

The data underlying this article will be shared on reasonable request to the corresponding author.

\section{Ethics approval and consent to participate}

The multi-country research design was approved by the Ethics Committee for the Social Sciences and Humanities of the University of Antwerp, as well as by the Ethics Committee for the Social Sciences of Ghent University. Each participant was asked to read an informed consent form and provide consent through checking a box.

\section{Consent for publication}

Not applicable.

\section{Competing interests}

The authors declare that there is no conflict of interest.

\section{Author details \\ ${ }^{1}$ Department of Family Medicine and Population Health, University of Antwerp, Doornstraat 331, 2610 Wilrijk, Belgium. ${ }^{2}$ Department of Sociology, University of Antwerp, Antwerp, Belgium. ' ${ }^{3}$ epartment of Sociology, Ghent University, Antwerp, Belgium. ${ }^{4}$ Social Epidemiology and Health Policy, University of Antwerp, Antwerp, Belgium.}

Received: 31 October 2020 Accepted: 20 December 2020 Published online: 07 January 2021

\section{References}

1. Johns Hopkins University. COVID-19 Map - Coronavirus Resource Center [Internet]. 2020 [cited 2020 Sep 29]. Available from: https://coronavirus.jhu. edu/map.html.
2. Sciensano. Covid-19: epidemiology [Internet]. [cited 2020 Aug 2]. Available from: https://covid-19.sciensano.be/nl/covid-19-epidemiologische-situatie.

3. Belgian Federal Government. Coronavirus: reinforced measures [Internet]. 2020 [cited 2020 Aug 5]. Available from: https://www.belgium.be/en/news/2 020/coronavirus_reinforced_measures.

4. Holmes EA, O'Connor RC, Perry VH, Tracey I, Wessely S, Arseneault L, et al. Multidisciplinary research priorities for the COVID-19 pandemic: a call for action for mental health science. Lancet Psychiatry. 2020;7(6):547-60 [cited 2020 Aug 17]. Available from: www.thelancet.com/psychiatry.

5. Lee J. Mental health effects of school closures during COVID-19. Lancet Child Adolesc Heal. 2020;4(6):421 [cited 2020 Aug 18]. Available from: www. thelancet.com/child-adolescent.

6. Viner RM, Russell SJ, Croker H, Packer J, Ward J, Stansfield C, et al. School closure and management practices during coronavirus outbreaks including COVID-19: a rapid systematic review [Internet], vol. 4: The Lancet Child and Adolescent Health. Elsevier B.V; 2020. p. 397-404. [cited 2020 Aug 18]. Available from: www.thelancet.com/child-adolescentVol.

7. O'Neill S, Auerbach R, Alonso J, ... WA-P, 2016 undefined. Mental disorders among college students in the World Health Organization World Mental Health Surveys. pure.ulster.ac.uk [Internet]. 2017 [cited 2020 Aug 18]; Available from: http://nrs.harvard.edu/urn-3:HUL.InstRepos:33461094.

8. Wörfel F, Gusy B, Lohmann K, Töpritz K, Kleiber D. Mental health problems among university students and the impact of structural conditions. J Public Health (Bangkok). 2016;24(2):125-33 [cited 2020 Aug 18]. Available from: http://link.springer.com/10.1007/s10389-015-0703-6.

9. Pierce M, Hope H, Ford T, Hatch S, Hotopf M, John A, et al. Mental health before and during the COVID-19 pandemic: a longitudinal probability sample survey of the UK population. Lancet Psychiatry. 2020;0(0) [cited 2020 Aug 18]. Available from: www.thelancet.com/psychiatryPublishedonline.

10. Kavčič T, Avsec A, Zager Kocjan G. Psychological Functioning of Slovene Adults during the COVID-19 Pandemic: Does Resilience Matter? Psychiatr Q. 2020:1-10 [cited 2020 Aug 21]. Available from: https://doi.org/10.1007/ s11126-020-09789-4.

11. Huang Y, Zhao N. Generalized anxiety disorder, depressive symptoms and sleep quality during COVID-19 outbreak in China: a web-based crosssectional survey. Psychiatry Res. 2020;288:112954.

12. Vijayaraghavan P, Singhal D. A Descriptive Study of Indian General Public's Psychological responses during COVID-19 Pandemic Lockdown Period in India. 2020; [cited 2020 Aug 17]. Available from: https://psyarxiv.com/jeksn/.

13. Kaparounaki CK, Patsali ME, Mousa DPV, Papadopoulou EVK, Papadopoulou KKK. Fountoulakis KN. University students' mental health amidst the COVID19 quarantine in Greece. Psychiatry Res. 2020;290:113111 Elsevier Ireland Ltd.

14. Cao W, Fang Z, Hou G, Han M, Xu X, Dong J, et al. The psychological impact of the COVID-19 epidemic on college students in China. Psychiatry Res. 2020;287:112934 [cited 2020 Aug 18]. Available from: https://linkinghub. elsevier.com/retrieve/pii/S0165178120305400.

15. Odriozola-González P, Planchuelo-Gómez Á, Irurtia MJ, de Luis-García R. Psychological effects of the COVID-19 outbreak and lockdown among students and workers of a Spanish university. Psychiatry Res. 2020;290:113108.

16. Brooks SK, Webster RK, Smith LE, Woodland L, Wessely S, Greenberg N, et al. The psychological impact of quarantine and how to reduce it: rapid review of the evidence. Lancet. 2020;395:912-20 Lancet Publishing Group. [cited 2020 Aug 17]. Available from: https://10.1016/.

17. Centre for population, family and health. The COVID-19 International Student Well-being Study [Internet]. [cited 2020 Aug 4]. Available from: https://www.uantwerpen.be/en/research-groups/centre-population-familyhealth/research2/covid-19-internation/.

18. van Buuren S, Groothuis-Oudshoorn K. mice: Multivariate Imputation by Chained Equations in R. J Stat Softw. 2011;45(3):1-67 Available from: https:// www.jstatsoft.org/v45/i03/.

19. Radloff LS. The CES-D scale. Appl Psychol Meas. 1977;1(3):385-401 Available from: http://journals.sagepub.com/doi/10.1177/014662167700100306.

20. Van de Velde S, Bracke P, Levecque K, Meuleman B. Gender differences in depression in 25 European countries after eliminating measurement bias in the CES-D 8. Soc Sci Res. 2010;39(3):396-404.

21. Hu L, Bentler PM. Cutoff criteria for fit indexes in covariance structure analysis: Conventional criteria versus new alternatives. Struct Equ Model A Multidiscip J. 1999;6(1):1-55 [cited 2019 Apr 25]. Available from: http://www. tandfonline.com/doi/abs/10.1080/10705519909540118.

22. Cerin E. Ways of unraveling how and why physical activity influences mental health through statistical mediation analyses. Ment Health Phys Act. 
2010;3(2):51-60 [cited 2019 May 16]. Available from: https://www. sciencedirect.com/science/article/pii/S1755296610000268.

23. Rosseel Y. lavaan: An R Package for Structural Equation Modeling. J Stat Softw. 2012;48(2) Available from: http:/www.jstatsoft.org/v48/i02/.

24. Jorgensen TD, Pornprasertmanit S, Schoemann AM, Rosseel Y. semTools: Useful tools for structural equation modeling. R package version 0.5-3. [Internet]. 2020 [cited 2020 Jun 1]. Available from: https:/cran.r-project.org/ package $=$ semTools.

25. Mazza C, Ricci E, Biondi S, Colasanti M, Ferracuti S, Napoli C, et al. A Nationwide Survey of Psychological Distress among Italian People during the COVID-19 Pandemic: Immediate Psychological Responses and Associated Factors. Int J Environ Res Public Health. 2020;17(9):3165 [cited 2020 Sep 8]. Available from: https:/www mdpi.com/1660-4601/17/9/3165.

26. Flemish government; department of education. Dataloep [Internet]. [cited 2020 Sep 3]. Available from: https://dataloep-publiek.vlaanderen.be/ QvAJAXZfc/notoolbar.htm?document=HO-Publiek\%2FPubliek Inschrijvingen_HO.qvw\&host=PubliekQVS\%40cwv100163\&anonymous=true. https://dataloep-publiek.vlaanderen.be/QvAJAXZfc/notoolbar. htm?document=HO-Publiek\%2FPubliek_l.

\section{Publisher's Note}

Springer Nature remains neutral with regard to jurisdictional claims in published maps and institutional affiliations.

Ready to submit your research? Choose BMC and benefit from:

- fast, convenient online submission

- thorough peer review by experienced researchers in your field

- rapid publication on acceptance

- support for research data, including large and complex data types

- gold Open Access which fosters wider collaboration and increased citations

- maximum visibility for your research: over $100 \mathrm{M}$ website views per year

At BMC, research is always in progress.

Learn more biomedcentral.com/submissions 